ARTICLE

Received 7 Nov 2013 | Accepted 11 Aug 2014 | Published 15 Sep $2014 \quad$ DOl: 10.1038/ncomms5959

\title{
Ultrafast quenching of electron-boson interaction and superconducting gap in a cuprate superconductor
}

\author{
Wentao Zhang1,2, Choongyu Hwang1,3, Christopher L. Smallwood ${ }^{1,2}$, Tristan L. Miller ${ }^{1,2}$, Gregory Affeldt ${ }^{1,2}$, \\ Koshi Kurashima ${ }^{4}$, Chris Jozwiak ${ }^{5}$, Hiroshi Eisaki ${ }^{6}$, Tadashi Adachi ${ }^{4,7}$, Yoji Koike ${ }^{4}$, Dung-Hai Lee ${ }^{2}$ \\ \& Alessandra Lanzara ${ }^{1,2}$
}

Ultrafast spectroscopy is an emerging technique with great promise in the study of quantum materials, as it makes it possible to track similarities and correlations that are not evident near equilibrium. Thus far, however, the way in which these processes modify the electron self-energy $-a$ fundamental quantity describing many-body interactions in a material-has been little discussed. Here we use time- and angle-resolved photoemission to directly measure the ultrafast response of self-energy to near-infrared photoexcitation in hightemperature cuprate superconductor. Below the critical temperature of the superconductor, ultrafast excitations trigger a synchronous decrease of electron self-energy and superconducting gap, culminating in a saturation in the weakening of electron-boson coupling when the superconducting gap is fully quenched. In contrast, electron-boson coupling is unresponsive to ultrafast excitations above the critical temperature of the superconductor and in the metallic state of a related material. These findings open a new pathway for studying transient self-energy and correlation effects in solids.

\footnotetext{
${ }^{1}$ Materials Sciences Division, Lawrence Berkeley National Laboratory, Berkeley, California 94720, USA. ${ }^{2}$ Department of Physics, University of California Berkeley, Berkeley, California 94720, USA. ${ }^{3}$ Department of Physics, Pusan National University, Busan 609-735, Republic of Korea. ${ }^{4}$ Department of Applied Physics, Tohoku University, Sendai 980-8579, Japan. ${ }^{5}$ Advanced Light Source, Lawrence Berkeley National Laboratory, Berkeley, California 94720, USA. ${ }^{6}$ Electronics and Photonics Research Institute, National Institute of Advanced Industrial Science and Technology, Ibaraki 305-8568, Japan. ${ }^{7}$ Department of Engineering and Applied Sciences, Sophia University, Tokyo 102-8554, Japan. Correspondence and requests for materials should be addressed to A.L. (email: alanzara@lbl.gov).
} 
T he dynamics of electrons and atoms interacting with intense and ultrashort optical pulses is one of the emerging fields in physics. Strong optical pulses have been used as powerful tools to measure the electron-phonon interaction in solids $s^{1,2}$, to investigate fundamental dynamical processes in semiconductors 3,4 and to modulate the lattice structure of solids by creating dynamical states with new properties ${ }^{5-8}$. These methods are particularly exciting in the context of correlated materials in which intense optical fields can drive a transition from an insulating to a metastable metallic phase ${ }^{9}$, can induce transient signatures of superconductivity ${ }^{10}$, can lead to anisotropic modulation of the electron-phonon coupling ${ }^{11}$ and can disentangle the different dynamics in governing the superconducting and pseudogap phase of cuprates ${ }^{12-15}$.

Despite the large amount of new physics revealed, most studies, thus far, use all-optical techniques that do not directly probe quasiparticles or carry any momentum information. As a consequence, the way fundamental quantities such as the electron self-energy and many-body interactions evolve outside equilibrium is often inferred indirectly. When probed in a timeresolved manner, these quantities have the potential to reveal insights on the microscopic properties of solids ${ }^{1,16}$. Recent developments in high-resolution time- and angle-resolved photoemission spectroscopy (trARPES) now make these studies possible. So far, however, most of the trARPES studies have focused on recombination dynamics of photo-induced quasiparticle population ${ }^{17-21}$ and gap dynamics $5,7,22,23$.

Here we present a study on the high-temperature cuprate superconductor $\mathrm{Bi}_{2} \mathrm{Sr}_{2} \mathrm{CaCu}_{2} \mathrm{O}_{8+\delta}(\mathrm{Bi} 2212)$, and compare it with metallic $\mathrm{Bi}_{1.76} \mathrm{~Pb}_{0.35} \mathrm{Sr}_{1.89} \mathrm{CuO}_{6+\delta}$ (Bi2201). In cuprate materials such as these, there is known to be a universal electron self-energy renormalization effect (a kink in the dispersion) that signifies the coupling of the electrons to bosons $\mathrm{s}^{2-28}$. However, whether this kink is related in any way to superconductivity is highly debated. Using trARPES, we tracked the temporal evolution of the electron self-energy renormalization and the superconducting gap after the system is perturbed by a femtosecond pump pulse. We found that, in superconducting Bi2212, the real part of the self-energy and the superconducting gap are markedly suppressed. Both effects also saturate at the same pump fluence. In contrast, in the normal state of Bi2212 and metallic heavily overdoped Bi2201, the suppression of the electron self-energy is far weaker. The results open a new avenue of investigation into self-energy and electron-boson interactions in solids.

Data were taken on an ultra-high resolution setup as previously described $^{29}$. In order to avoid the effects of photon-induced electric fields ${ }^{16}$, we limit our study to relatively low fluence (between 4 and $24 \mu \mathrm{J} \mathrm{cm}^{-2}$ or between 0.004 and $0.009 \mathrm{~V} / a_{0}$ ) and long time delay ( $\geq 300 \mathrm{fs}$ ). However, the fluence used in this study is still high enough to drive the full closure of the superconducting gap on the Fermi $\operatorname{arc}^{23}$.

\section{Results}

Electronic dispersion. Figure 1a shows the equilibrium $(t=$ $-1 \mathrm{ps})$ and non-equilibrium ( $t=1$ and $10 \mathrm{ps}$ ) ARPES intensity as a function of energy and momentum for a nearly optimally doped sample measured with pump fluence $24 \mu \mathrm{J} \mathrm{cm}^{-2}$ at $17 \mathrm{~K}$, far below $T_{c}$, along the nodal direction. The equilibrium spectrum shows the widely studied renormalization kink at $\sim 70 \mathrm{meV}$ (refs 24-27). Upon pumping, at a delay time of $1 \mathrm{ps}$, a clear loss of spectral weight can be observed, which is mainly confined between the Fermi energy and the kink energy ${ }^{18}$. It takes $\sim 10 \mathrm{ps}$ for the transient spectra to recover back to the equilibrium state. The effect of laser pumping on the dispersion is shown in Fig. 1b, where the equilibrium (black solid line) and transient (red solid
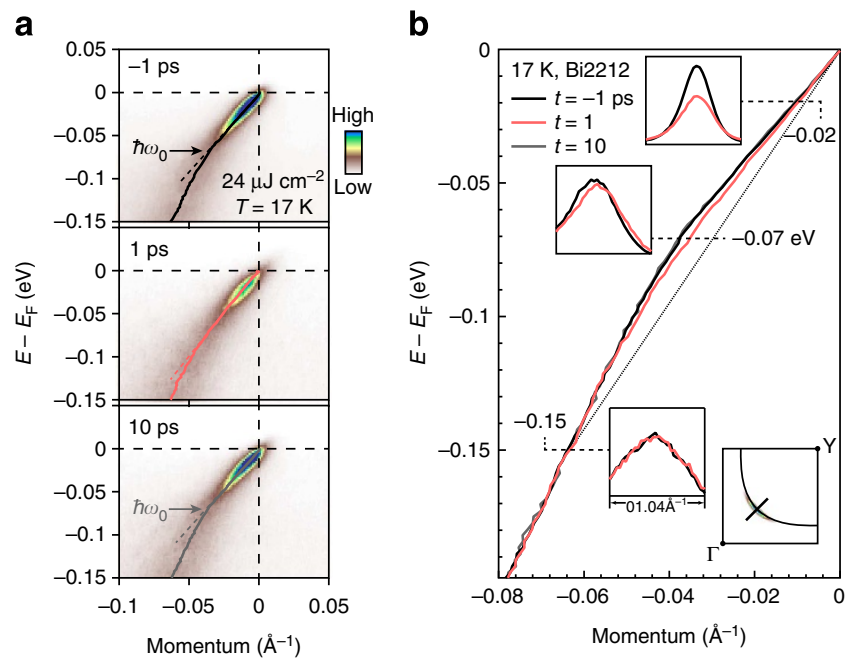

Figure 1 | Time-resolved spectra on a nearly optimally doped Bi2212. (a) Equilibrium (before pumping, $t=-1 \mathrm{ps}$ ) and transient (after pumping, $t=1$ and $10 \mathrm{ps}$ ) photoelectron intensity (represented by false colour) as a function of energy and momentum measured along a nodal cut for a pump fluence of $24 \mu \mathrm{Jm}^{-2}$. The bold solid black lines are the momentum distribution curve (MDC) dispersions at the corresponding delay time. The arrows mark the position of the kink at $\hbar \omega_{0} \sim 70 \mathrm{meV}$. (b) MDC dispersions for different delay times $(-1,1$ and $10 \mathrm{ps})$. Insets show comparisons of MDCs before pumping ( $-1 \mathrm{ps}$ ) and after pumping (1ps) for a series of binding energies $(-0.15,-0.07$ and $-0.02 \mathrm{eV})$.

line) dispersions are compared. The dispersions are extracted in the standard way by fitting momentum distribution curves (MDCs) to a Lorentzian functional form ${ }^{30}$. The comparison between the dispersion curve after a long delay time $(t=10 \mathrm{ps}$; grey line) with the equilibrium curve provides an estimate of our error bars. The most obvious pump-induced change occurs near the kink energy, as shown by the shift of MDC peak position in this energy range, in contrast to the MDCs near the Fermi level or at much higher binding energies, where the shift is negligible (see inset). Specifically, for delay time $t=1 \mathrm{ps}$, the Fermi velocity increases by $0.13 \mathrm{eV} \AA$ (equilibrium $1.87 \mathrm{eV} \AA$ ) at binding energy below the kink energy and remains approximately unchanged above, resulting in an apparent softening of the kink strength (such as coupling strength) as pumping is turned on. The Fermi velocity is extracted from the slope in the dispersion between $\sim 70 \mathrm{meV}$ and $E_{\mathrm{F}}$, as $v=\mathrm{d} E / \mathrm{d} k^{31}$.

Self-energy changes below $T_{c}$. The differences between equilibrium and transient spectra can be analysed by extracting the electron self-energy $\Sigma=\Sigma^{\prime}+i \Sigma^{\prime \prime}$, shown in Fig. 2 . To extract the effective real part of the self-energy at different delay time, we took measured dispersions and subtracted featureless linear bare bands with the same velocity at each delay time (see, for example, the dotted line in Fig. 1b). Such techniques are commonly used to analyse the electron-boson interaction at equilibrium ${ }^{24,30}$. At equilibrium (black dots) $\Sigma^{\prime}$ is reminiscent of a spectrum of modes, as previously reported ${ }^{26,27,32,33}$, and its maximum is at the $70 \mathrm{meV}$ kink position. The most significant pump-induced effect is the suppression of $\Sigma^{\prime}$ in the proximity of the kink energy (between 40 and $90 \mathrm{meV}$; Fig. $2 \mathrm{a}$, red dots), in line with a softening of the coupling strength, as shown in Fig. 1. This suppression intensifies as fluence increases, and eventually saturates when superconductivity is completely suppressed (Fig. 3), as we will discuss later. Within our resolution, we observe no pump-induced energy shift in the peak position of $\Sigma^{\prime}$ (ref. 34). In the same 
a

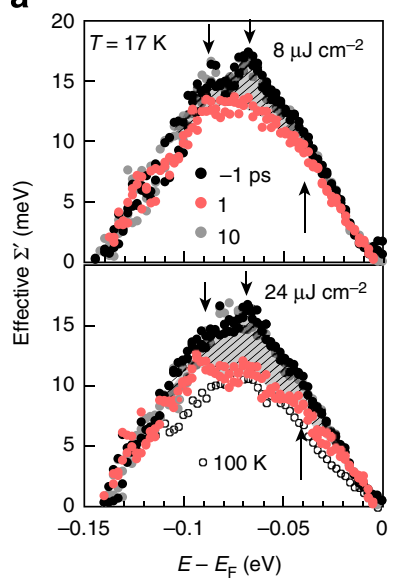

b

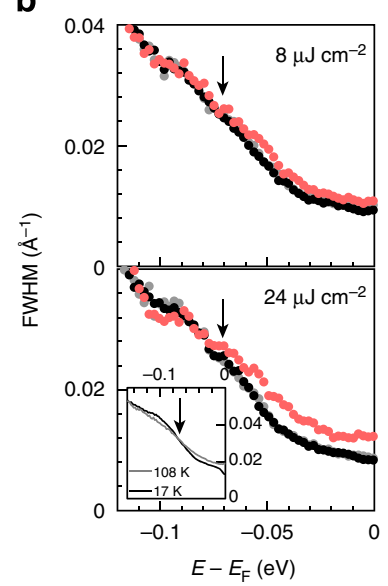

C

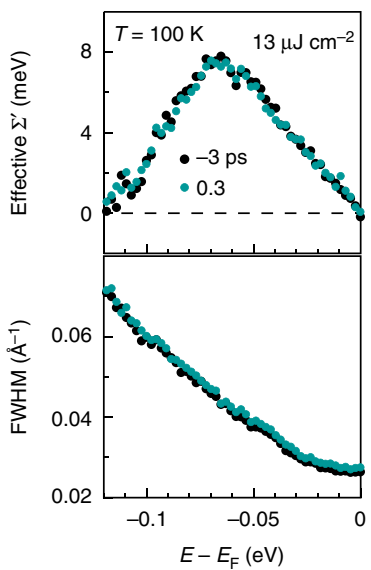

Figure 2 | Equilibrium and transient nodal electron self-energies. (a) Real part of electron self-energy $\left(\Sigma^{\prime}\right)$ at different delay times $(t=-1,1$ and 10 ps) for pump fluences 8 and $24 \mu \mathrm{Jm}^{-2}$ measured at $17 \mathrm{~K}$ (below $T_{\mathrm{c}}$ ). $\Sigma^{\prime}$ measured at equilibrium temperature $100 \mathrm{~K}$ (above $T_{\mathrm{c}}$ ) is plotted in the lowest panel for comparison. (b) The corresponding MDC width as a function of energies. The black arrows mark the energy $\sim \hbar \omega_{0}$ where non-equilibrium and equilibrium MDC width separate with each other. Inset in the lowest panel shows non-equilibrium MDC width at 17 and $108 \mathrm{~K}$ (ref. 32 ). (c) $\Sigma^{\prime}$ and MDC width, similar to that shown in $\mathbf{a}, \mathbf{b}$ but measured in the normal state with pump fluence $13 \mu \mathrm{J} \mathrm{cm}^{-2}$, at an equilibrium temperature of $100 \mathrm{~K}$.

a
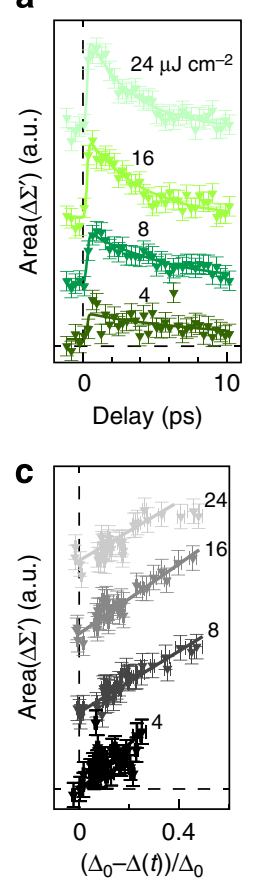

b


e

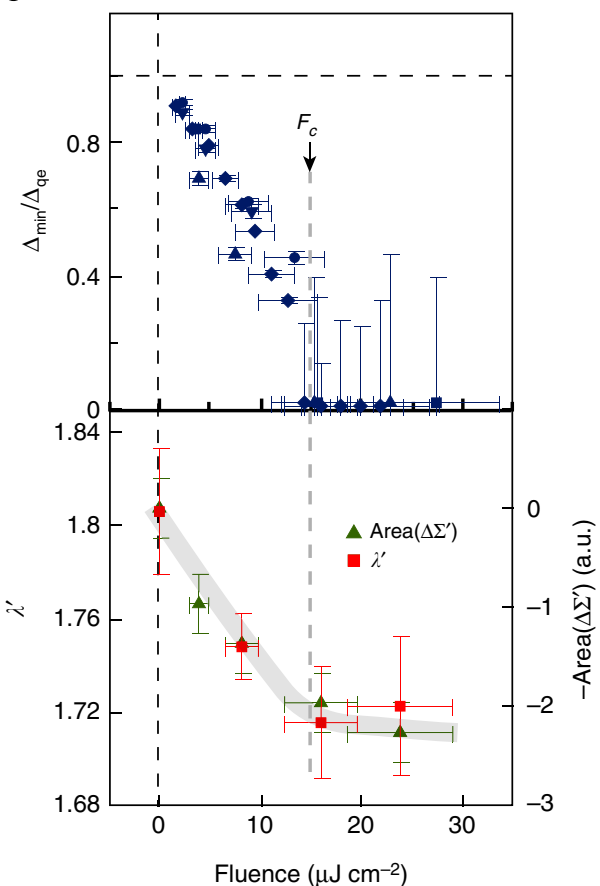

Figure 3 | Dynamics of pump-induced change. (a) Pump-induced change of nodal $\Sigma^{\prime}$ (vertically offset for clarity) as a function of delay time for different fluences. The error bars of $\operatorname{Area}\left(\Delta \Sigma^{\prime}\right)$ are estimated by the maximum difference of $\operatorname{Area}\left(\Delta \Sigma^{\prime}\right)$ among the measurements in equilibrium state. (b) Gap versus pump-probe delay time for an off-nodal cut (inset) at different fluences. The changes of energy gap are normalized to the maximum gap. (c) Pumpinduced change of the $\Sigma^{\prime}$ (offset for clarity) as a function of the energy gap at each delay time as extracted from $\mathbf{a}, \mathbf{b}$ for four different fluences. Because of the limited energy resolution ( $23 \mathrm{meV}$, comparable with the equilibrium gap size), only energy gap changes $<50 \%$ are reliable and shown. (d) The recovery rate of nodal $\Delta \Sigma^{\prime}$ and the nodal coupling strength $\lambda^{\prime}$ as a function of fluence. (e) The pump-induced non-equilibrium minimum energy gap, maximum changes of $\operatorname{Area}\left(\Delta \Sigma^{\prime}\right)$ and electron-boson coupling strength at $70 \mathrm{meV}$ as a function of pump fluence. The coupling strength is given by $\lambda^{\prime}=v_{0} / v_{\mathrm{F}}$ as functions of pump fluence $\left(v_{\mathrm{O}}\left(v_{\mathrm{F}}\right)\right.$, bare (Fermi) velocity below (above) the kink energy). The error bars of the change of $\Sigma^{\prime}$ and $\lambda^{\prime}$ are absolute maximum variations before $t=0$, and the others are s.d. from fitting.

figure (bottom panel of Fig. 2a), we directly compare the equilibrium $\Sigma^{\prime}$ at $100 \mathrm{~K}$ with the pump-induced $\Sigma^{\prime}$ at a similar electronic temperature as measured from the width of the Fermi edge ${ }^{17,18,23}$. While the effect of temperature on the equilibrium
$\Sigma^{\prime}$ extends over the entire energy range, the effect of pumping is smaller overall and is mainly confined within the kink energy (see also Supplementary Fig. 1). This suggests that optical pumping induces an effect beyond increasing the temperature. 
In Fig. 2b, we show the imaginary part of the self-energy, $\Sigma^{\prime \prime}$, that is proportional to the full-width at half maximum of the MDCs. In agreement with $\Sigma^{\prime}$, the main pump-induced change is confined between the kink energy and the Fermi level (vertical black arrow) and increases as fluence increases. This is in contrast to thermal effects, where the temperature causes a change of $\Sigma^{\prime \prime}$ over the entire energy window (see inset in Fig. $2 b$ and Supplementary Fig. 1). In Fig. 2c, we show the comparison of equilibrium and transient $\Sigma^{\prime}$ and $\Sigma^{\prime \prime}$ at equilibrium temperature $100 \mathrm{~K}$, above $T_{c}$. In sharp contrast with the low-temperature behaviour, the pump-induced changes of the self-energy are negligible in the normal state up to the highest applied fluence $24 \mu \mathrm{J} \mathrm{cm}^{-2}$ (we note, however, that this might no longer be valid at very high fluences, which would substantially affect the entire band structure ${ }^{16,35}$. These results might suggest that the pumpinduced changes of self-energy are sensitive to the presence of superconductivity and are not induced by trivial thermal broadening effects.

We note that the absence of a shift in the kink energy, when the superconducting gap closes, suggests that the electron-boson interaction falls beyond the standard Migdal-Eliashberg theory for superconductivity as also suggested by equilibrium ARPES experiments ${ }^{36,37}$. Indeed, within this standard theory, the energy dispersion has a square-root divergence at energy $\Delta_{0}+\Omega$ in $\Sigma^{\prime}(\omega)\left(\Omega\right.$ is the boson energy and $\Delta_{0}$ is the superconducting gap at zero temperature $)^{38}$. Therefore, when the pump drives the superconducting gap to zero, one expects a shift of the kink energy and of $\Sigma^{\prime}$ towards lower binding energy by the magnitude of the superconducting gap, even for the nodal $\mathrm{cut}^{39}$.

Self-energy versus superconducting gap. To further investigate this matter, we utilize the unique advantage of trARPES by simultaneously monitoring the pump-induced changes in both the electron self-energy and the superconducting gap. Figure 3 compares the pump-induced change in $\Sigma^{\prime}$ and the non-equilibrium superconducting gap. The latter is measured for a cut on the Fermi arc (see inset of Fig. 3b). Energy gaps at each delay time are obtained by fitting symmetrized energy distribution curves to an energy-resolution-convolved phenomenological BCS model ${ }^{40}$, which is widely used in characterizing the energy gap in
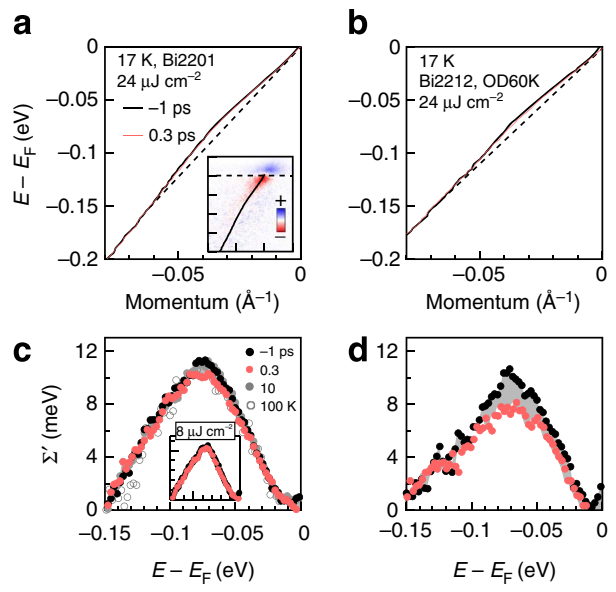

cuprates $^{23,41}$ (see also Supplementary Fig. 2). The temporal evolution of the area of $\Delta \Sigma^{\prime}$ (hatched area in Fig. 2a) and the superconducting gap for different fluences are shown in panels a and b of Fig. 2, respectively. In agreement with Fig. 2, $\Sigma^{\prime}$ is weakly affected at low fluences and shows substantially slower initial recovery rate $\left(0.1 \mathrm{ps}^{-1}\right)$ than at higher fluences $\left(0.32 \mathrm{ps}^{-1}\right.$; Fig. 3d). Similarly, pumping only weakly affects the superconducting gap at low fluence (bottom measurements in Fig. 3b) and eventually drives it to a full closure at high fluences (top measurements) ${ }^{20,23}$. A similar fluence dependence is also observed for the Fermi velocity (Supplementary Fig. 3). The pump-induced change in the self-energy and the non-equilibrium superconducting gap at each delay time are plotted in Fig. 3c for several fluences, showing an unexpected linear relation for all fluences. At the highest fluence, a small deviation from linearity is observed, possibly because of additional broadening of the spectra, causing an underestimate of the superconducting gap.

In Fig. 3e, we show the fluence dependence of the maximal nearnodal gap shift (Fig. 3b) and compare it with the area of the maximal self-energy change (from Fig. 3a) and the coupling constant. In a simple electron-boson coupling model ${ }^{42}$, the coupling strength $\lambda$ is directly related to the bare and dressed Fermi velocities according to $\lambda^{\prime} \equiv \lambda+1=v_{\mathrm{F}} / v_{0}$. Alternatively, the change in $\lambda$ can be approximated by the integral of the self-energy change between equilibrium and pumped values (see hatched areas in Fig. 2) because near the Fermi energy $\lambda=\lim _{\omega \rightarrow 0} \partial \Sigma^{\prime} / \partial \omega$, namely, $\quad \Sigma^{\prime}(\omega) \approx \lambda \omega$, thus $\int_{0}^{\omega_{c}} \Delta \Sigma^{\prime}(\omega) \mathrm{d} \omega \approx \Delta \lambda \omega_{\mathrm{c}}^{2} / 2$. Both characterization methods are shown (for more details, see also Supplementary Fig. 3). Interestingly, as we drive the superconducting gap to a gradual melting by increasing the excitation density, we find that the electron-boson interaction decreases in a similar fashion and eventually saturates (see also Supplementary Fig. 4) when the superconducting gap is fully quenched. The saturation effect is in contrast with thermal broadening, where continuous smearing of the kink occurs as the temperature gradually increases ${ }^{32}$.

Figure $3 \mathrm{~d}$ shows the recovery rate of the integrated self-energy change (from Fig. 3a) and $\lambda^{\prime}$ (Supplementary Fig. 3). In the lowfluence regime, we find that both of these rates increase linearly with fluence in a similar fashion to the bimolecular recombination of non-equillibrium quasiparticles ${ }^{20,43}$. As the fluence

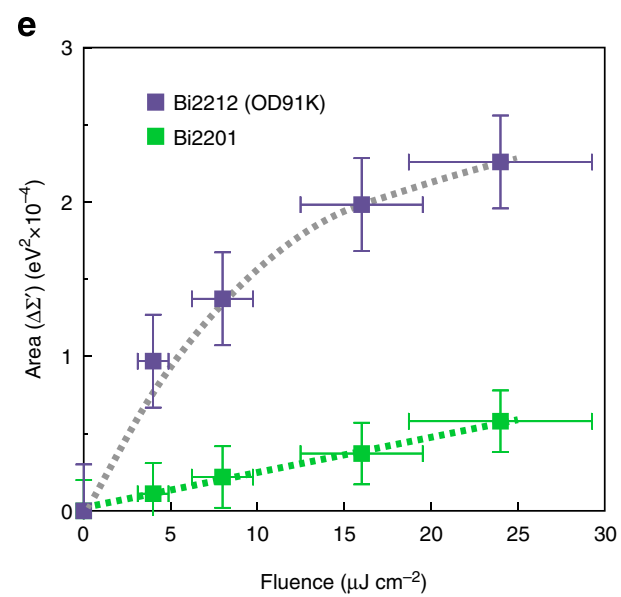

Figure 4 | Results on a heavily overdoped Bi2201 and an overdoped Bi2212. (a,b) MDC dispersions of equilibrium ( $t=-1 \mathrm{ps})$ and non-equilibrium $(t=0.3 \mathrm{ps})$ states, for a pump fluence of $24 \mu \mathrm{J} \mathrm{cm}{ }^{-2}$ for the overdoped Bi2201 and the overdoped Bi2212, respectively. False-coloured inset in a shows the difference between equilibrium ( $t=-1 \mathrm{ps})$ and non-equilibrium $(t=0.3 \mathrm{ps})$ raw spectral image. (c,d) Real part of the electron self-energy $\left(\Sigma^{\prime}\right)$ at different delay time $\left(t=-1,0.3\right.$ and $10 \mathrm{ps}$ ) for pump fluence $24 \mu \mathrm{J} \mathrm{cm}{ }^{-2}$ for the overdoped Bi2201 and the overdoped Bi2212, respectively. The equilibrium $\Sigma^{\prime}$ measured at $100 \mathrm{~K}$ on the overdoped Bi2201 is represented by empty circles for comparison in c. The inset in c shows $\Sigma^{\prime}$ spectra for a pump fluence of $8 \mu \mathrm{Jm}^{-2}$. (e) Fluence dependence of the Area $\left(\Sigma^{\prime}\right)$ for the optimally doped Bi2212 and heavily overdoped Bi2201. The bold lines are guides to the eye. The error bars in $\mathbf{d}$ are absolute maximum variations before $t=0$. 
approaches the critical fluence, both rates saturate, marking the onset of different recombination processes, a behaviour that appears to be dictated by the closing of the superconducting gap at $F_{c}$. The behaviour is also consistent with previous reports of non-equilibrium quasiparticle recombination in an optimally doped and underdoped Bi2212 (refs 20,21), and with a timeresolved optical reflectivity study ${ }^{44}$.

Self-energy changes above. To better understand the nature of the reported pump-induced change in the self-energy of Bi2212 superconductor, in Fig. 4, we study the effect of pumping on the electron-boson coupling in a metal in the same low-fluence regime and at the same low temperature. To make a meaningful comparison with Bi2212, we report data for an heavily overdoped Bi2201 $\left(T_{\mathrm{c}}<2 \mathrm{~K}\right)$. Bi2201 has very similar crystallographic and electronic structure to Bi2212, and an only slightly weaker $70 \mathrm{meV}$ dispersion kink ${ }^{25}$. Moreover, Bi2201 can be grown in the heavily overdoped regime, with $T_{c}$ lower than a few Kelvin, making it possible for us to study its normal state at $17 \mathrm{~K}$, the same temperature as the study of optimally doped Bi2212.

Figure 4a shows the pump-induced changes in the electronic dispersion of heavily overdoped metallic Bi2201 along the nodal direction. Although qualitatively the pump-induced changes of spectral intensity are similar to those in superconducting optimally doped Bi2212 (ref. 18), there are significant differences between the two. Despite the apparent kink feature, the pump-induced changes in $\Sigma^{\prime}$ are negligible at lower fluences (inset of Fig. 4c), and show a very small increase with fluence over the entire range. Note that, consistent with the notion that the critical fluence marks the threshold above which all Cooper pairs are broken ${ }^{21}$, no critical fluence is observed in the $\Sigma^{\prime}$ in the normal state of this heavily overdoped sample (Fig. 4e). This difference is not likely owing to the weaker kink in Bi2201. Indeed, at the highest fluence (Fig. 4c), the change of $\Sigma^{\prime}$ is still at least a factor of 2 smaller than the corresponding change observed in overdoped Bi2212-displayed in Fig. 4d-despite the comparable kink strength. The change of $\Sigma^{\prime}$ in Bi2201 is a factor of 4 smaller than optimally doped Bi2212, in which the kink strength is larger only by a factor of $40 \%$ (see Fig. $4 \mathrm{e}$ and the coupling strength in Supplementary Fig. 5). In contrast to Bi2212, the pump-induced changes in Bi2201 can be accounted for by thermal smearing of the dispersion with temperature ${ }^{33,45}$, as shown by the comparison of equilibrium and transient selfenergy at an equilibrium temperature of $100 \mathrm{~K}$. We caution that these considerations apply to the low pump fluence regime. This behaviour is also consistent with the expected behaviour for a metal in which temperature smears the kink feature and reduces the magnitude of the maximum of $\Sigma^{\prime}$ without any change in the electron-boson coupling ${ }^{38}$.

\section{Discussion}

The effects observed in this paper cannot be simply explained by transient heating. Indeed, a comparison at similar temperatures between the temperature dependence of the transient self-energy and the equilibrium self-energy shows quite different trends. At equilibrium the temperature-dependent self-energy changes over a broad range of energies ${ }^{32}$, in contrast to the transient selfenergy, where the changes are confined to the vicinity of the kink energy. In addition, the equilibrium self-energy gradually changes as the equilibrium temperature increases, and the effect persists even above $T_{c}$. In contrast, the pump-induced change in the selfenergy saturates at the critical fluence and does not show any change above $T_{\mathrm{c}}$. Moreover, the temperature dependence of the density of the initial non-equilibrium quasiparticles population is not proportional to the inverse of the specific heat ${ }^{21}$, as expected in the case of thermal dynamics. Finally, the observation that bimolecular recombination dominates quasiparticle scattering processes in the low-fluence regime further supports the nonthermal nature of the reported effects ${ }^{20,43}$.

These observations, together with the negligible pump-induced effect in the normal state and in a metallic compound (Fig. 4) validate the intrinsic nature of the pump-induced phenomena reported here. Whether these effects are due to a change of the electron-boson coupling constant as in an equilibrium picture or are a consequence of photo-induced delocalization of charge that leads to a reduction of the Jahn-Teller effect ${ }^{46,47}$ and to an apparent softening of the electron-boson interaction, they suggest the exciting possibility that the effects responsible for the selfenergy renormalization are an important booster of a large pairing gap $^{26-30}$. trARPES experiments in which one can coherently pump a specific phonon mode may provide fundamental new insights into this piece of the puzzle.

\section{Methods}

Time-resolved ARPES. In our trARPES experiments ${ }^{18,20,29}$, an infrared pump laser pulse $(h v=1.48 \mathrm{eV})$ drives the sample into a non-equilibrium state. Subsequently, an ultraviolet probe laser pulse $(5.93 \mathrm{eV})$ photoemits electrons that are captured by a hemispherical analyser in an ARPES setup. The system is equipped with a Phoibos 150-mm hemispherical electron energy analyser (SPECS). The spot size (full-width at half maximum) of pump and probe beams are $\sim 100$ and $\sim 50 \mu \mathrm{m}$, respectively, and the pump fluence is calculated using the same method as previously reported ${ }^{20}$. The repetition rate of the pump and probe beams used in the measurements is $543 \mathrm{kHz}$. Time resolution is achieved by varying the delay time $(t)$ between the probe and pump pulses. For $t<0$, the probe pulse arrives before the pump pulse, and hence corresponds to an equilibrium measurement. For $t>0$, the probe pulse arrives after the pump pulse, and hence corresponds to a nonequilibrium measurement. The zero delay and time resolution are determined by a cross-correlation of pump and probe pulses measured on hot electrons of polycrystalline gold integrated in a $0.4-\mathrm{eV}$ kinetic energy window centred at $1.0 \mathrm{eV}$ above the Fermi energy. The total energy resolution is $\sim 22 \mathrm{meV}$, time resolution is $\sim 310$ fs (Supplementary Fig. 6) and the momentum resolution is $\sim 0.003 \AA^{-1}$ at nodal point at the Fermi energy. In the electron self-energy analysis, the LucyRichardson iterative deconvolution method is applied to mitigate the effect of the energy resolution ${ }^{48}$.

Samples. Nearly optimally doped $\mathrm{Bi}_{2} \mathrm{Sr}_{2} \mathrm{CaCu}_{2} \mathrm{O}_{8+\delta}\left(T_{\mathrm{c}}=91 \mathrm{~K}\right)$ and heavily overdoped $\mathrm{Bi}_{1.76} \mathrm{~Pb}_{0.35} \mathrm{Sr}_{1.89} \mathrm{CuO}_{6+\delta}$ single crystals were grown by the travelling solvent floating zone method. The overdoped Bi2212 sample $\left(T_{\mathrm{c}}=60 \mathrm{~K}\right)$ and heavily overdoped Bi2201 sample $\left(T_{\mathrm{c}}<2 \mathrm{~K}\right)$ were obtained by annealing the asgrown optimally doped $\mathrm{Bi} 2212$ and $\mathrm{Bi} 2201$ in high pressure oxygen. The $T_{\mathrm{c}}$ values of the samples were characterized by superconducting quantum interference measurements. Samples were cleaved in situ in ultra-high vacuum with a base pressure $<5 \times 10^{-11}$ Torr.

\section{References}

1. Allen, P. B. Theory of thermal relaxation of electrons in metals. Phys. Rev. Lett 59, 1460-1463 (1987).

2. Brorson, S. D. et al. Femtosecond room-temperature measurement of the electron-phonon coupling constant $\lambda$ in metallic superconductors. Phys. Rev. Lett. 64, 2172-2175 (1990)

3. Gupta, S. Carrier Dynamics in III-V Materials and Hetero-Structures Studied by Ultrafast Laser Techniques (Semiconductors, Optoelectronics, Group III V Materials) (University of Michigan, 1992).

4. Ulbricht, R., Hendry, E., Shan, J., Heinz, T. F. \& Bonn, M. Carrier dynamics in semiconductors studied with time-resolved terahertz spectroscopy. Rev. Mod. Phys. 83, 543-586 (2011).

5. Schmitt, F. et al. Transient electronic structure and melting of a charge density wave in tbte3. Science 321, 1649-1652 (2008).

6. Tomeljak, A. et al. Dynamics of photoinduced charge-density-wave to metal phase transition in $\mathrm{K}_{0.3} \mathrm{MoO}_{3}$. Phys. Rev. Lett. 102, 066404 (2009).

7. Rohwer, T. et al. Collapse of long-range charge order tracked by time-resolved photoemission at high momenta. Nature 471, 490-493 (2011).

8. Mizoguchi, K., Morishita, R. \& Oohata, G. Generation of coherent phonons in a CdTe single crystal using an ultrafast two-phonon laser-excitation process. Phys. Rev. Lett. 110, 077402 (2013).

9. Rini, M. et al. Photoinduced phase transition in $V \mathrm{O}_{2}$ nanocrystals: ultrafast control of surface-plasmon resonance. Opt. Lett. 30, 558-560 (2005).

10. $\mathrm{Hu}, \mathrm{W}$. et al. Optically enhanced coherent transport in $\mathrm{Y} \mathrm{Ba}_{2} \mathrm{Cu}_{3} \mathrm{O}_{6.5}$ by ultrafast redistribution of interlayer coupling. Nat. Mater. 13, 705-711 (2014) 
11. Carbone, F., Yang, D.-S., Giannini, E. \& Zewail, A. H. Direct role of structural dynamics in electron-lattice coupling of superconducting cuprates. Proc. Natl Acad. Sci. USA 105, 20161-20166 (2008).

12. Demsar, J., Podobnik, B., Kabanov, V. V., Wolf, T. \& Mihailovic, D. Superconducting gap $\delta_{c}$, the pseudogap $\delta_{p}$, and pair fluctuations above $T_{c}$ in overdoped $\mathrm{Y}_{1-x} \mathrm{Ca}_{x} \mathrm{Ba}_{2} \mathrm{Cu}_{3} \mathrm{O}_{7-\delta}$ from femtosecond time-domain spectroscopy. Phys. Rev. Lett. 82, 4918-4921 (1999).

13. Kaindl, R. A. et al. Ultrafast mid-infrared response of $\mathrm{Y} \mathrm{Ba}_{2} \mathrm{Cu}_{3} \mathrm{O}_{7-\delta}$. Science 287, 470-473 (2000).

14. Hinton, J. P. et al. New collective mode in $\mathrm{YBa}_{2} \mathrm{Cu}_{3} \mathrm{O}_{6+x}$ observed by time-domain reflectometry. Phys. Rev. B 88, 060508 (2013).

15. Coslovich, G. et al. Competition between the pseudogap and superconducting states of $\mathrm{Bi}_{2} \mathrm{Sr}_{2} \mathrm{Ca}_{0.92} \mathrm{Y}_{0.08} \mathrm{Cu}_{2} \mathrm{O}_{8+\delta}$ single crystals revealed by ultrafast broadband optical reflectivity. Phys. Rev. Lett. 110, 107003 (2013).

16. Sentef, M. et al. Examining electron-boson coupling using time-resolved spectroscopy. Phys. Rev. X 3, 041033 (2013).

17. Perfetti, L. et al. Ultrafast electron relaxation in superconducting $\mathrm{Bi}_{2} \mathrm{Sr}_{2} \mathrm{CaCu}_{2} \mathrm{O}_{8+\delta}$ by time-resolved photoelectron spectroscopy. Phys. Rev. Lett. 99, 197001 (2007).

18. Graf, J. et al. Nodal quasiparticle meltdown in ultrahigh-resolution pumpprobe angle-resolved photoemission. Nat. Phys. 7, 805-809 (2011).

19. Cortés, R. et al. Momentum-resolved ultrafast electron dynamics in superconducting $\mathrm{Bi}_{2} \mathrm{Sr}_{2} \mathrm{CaCu}_{2} \mathrm{O}_{8+\delta}$. Phys. Rev. Lett. 107, 097002 (2011).

20. Smallwood, C. L. et al. Tracking Cooper pairs in a cuprate superconductor by ultrafast angle-resolved photoemission. Science 336, 1137-1139 (2012).

21. Zhang, W. et al. Signatures of superconductivity and pseudogap formation in nonequilibrium nodal quasiparticles revealed by ultrafast angle-resolved photoemission. Phys. Rev. B 88, 245132 (2013).

22. Brouet, V. et al. Ultrafast filling of an electronic pseudogap in an incommensurate crystal. Phys. Rev. B 87, 041106 (2013).

23. Smallwood, C. L. et al. Time- and momentum-resolved gap dynamics in $\mathrm{Bi}_{2} \mathrm{Sr}_{2} \mathrm{CaCu}_{2} \mathrm{O}_{8}$. Phys. Rev. B 89, 115126 (2014).

24. Bogdanov, P. V. et al. Evidence for an energy scale for quasiparticle dispersion in $\mathrm{Bi}_{2} \mathrm{Sr}_{2} \mathrm{CaCu}_{2} \mathrm{O}_{8}$. Phys. Rev. Lett. 85, 2581-2584 (2000).

25. Lanzara, A. et al. Evidence for ubiquitous strong electron-phonon coupling in high-temperature superconductors. Nature 412, 510-514 (2001).

26. Kaminski, A. et al. Renormalization of spectral line shape and dispersion below $T_{c}$ in $\mathrm{Bi}_{2} \mathrm{Sr}_{2} \mathrm{CaCu}_{2} \mathrm{O}_{8+\delta}$. Phys. Rev. Lett. 86, 1070-1073 (2001).

27. Johnson, P. D. et al. Doping and temperature dependence of the mass enhancement observed in the cuprate $\mathrm{Bi}_{2} \mathrm{Sr}_{2} \mathrm{CaCu}_{2} \mathrm{O}_{8+\delta}$. Phys. Rev. Lett. 87, 177007 (2001)

28. Gweon, G.-H. et al. An unusual isotope effect in a high-transition-temperature superconductor. Nature 430, 187-190 (2004).

29. Smallwood, C. L., Jozwiak, C., Zhang, W. T. \& Lanzara, A. An ultrafast angle-resolved photoemission apparatus for measuring complex materials. Rev. Sci. Instrum. 83, 123904 (2012).

30. Valla, T., Fedorov, A. V., Johnson, P. D. \& Hulbert, S. L. Many-body effects in angle-resolved photoemission: quasiparticle energy and lifetime of a $\mathrm{Mo}(110)$ surface state. Phys. Rev. Lett. 83, 2085-2088 (1999).

31. Ashcroft, N. \& Mermin, N. Solid State Physics (Holt, Rinehart and Winston, 1976).

32. Zhang, W. T. et al. Identification of a new form of electron coupling in the $\mathrm{Bi}_{2} \mathrm{Sr}_{2} \mathrm{CaCu}_{2} \mathrm{O}_{8}$ superconductor by laser-based angle-resolved photoemission spectroscopy. Phys. Rev. Lett. 100, 107002 (2008).

33. Lee, W. S. et al. Superconductivity-induced self-energy evolution of the nodal electron of optimally doped $\mathrm{Bi}_{2} \mathrm{Sr}_{2} \mathrm{Ca}_{0.92} \mathrm{Y}_{0.08} \mathrm{Cu}_{2} \mathrm{O}_{8+\delta}$. Phys. Rev. B 77, 140504 (2008).

34. Kulić, M. L. \& Dolgov, O. V. Dominance of the electron-phonon interaction with forward scattering peak in high- $T_{c}$ superconductors: theoretical explanation of the ARPES kink. Phys. Rev. B 71, 092505 (2005).

35. Rameau, J. D. et al. Photoinduced changes in the cuprate electronic structure revealed by femtosecond time- and angle-resolved photoemission. Phys. Rev. B 89, 115115 (2014).
36. He, J. et al. Coexistence of two sharp-mode couplings and their unusual momentum dependence in the superconducting state of $\mathrm{Bi}_{2} \mathrm{Sr}_{2} \mathrm{CaCu}_{2} \mathrm{O}_{8}+\delta$ revealed by laser-based angle-resolved photoemission. Phys. Rev. Lett. 111, 107005 (2013).

37. Plumb, N. C. et al. Large momentum-dependence of the main dispersion kink in the high- $\mathrm{T}_{c}$ superconductor $\mathrm{Bi}_{2} \mathrm{Sr}_{2} \mathrm{CaCu}_{2} \mathrm{O}_{8+\delta}$. New J. Phys. 15, 113004 (2013).

38. Scalapino, D. J. The electron-phonon interaction and strong-coupling superconductors, superconductivity. Superconductivity Vol. I (CRC Press, 1969).

39. Sandvik, A. W., Scalapino, D. J. \& Bickers, N. E. Effect of an electron-phonon interaction on the one-electron spectral weight of a $d$-wave superconductor. Phys. Rev. B 69, 094523 (2004).

40. Norman, M. R., Randeria, M., Ding, H. \& Campuzano, J. C. Phenomenology of the low-energy spectral function in high- $T_{c}$ superconductors. Phys. Rev. B 57, R11093-R11096 (1998).

41. Lee, W. S. et al. Abrupt onset of a second energy gap at the superconducting transition of underdoped Bi2212. Nature 450, 81-84 (2007).

42. Park, S. R. et al. Angle-resolved photoemission spectroscopy of electron-doped cuprate superconductors: isotropic electron-phonon coupling. Phys. Rev. Lett. 101, 117006 (2008)

43. Gedik, N. et al. Single-quasiparticle stability and quasiparticle-pair decay in $Y$ $\mathrm{Ba}_{2} \mathrm{Cu}_{3} \mathrm{O}_{6.5}$. Phys. Rev. B 70, 014504 (2004).

44. Coslovich, G. et al. Evidence for a photoinduced nonthermal superconductingto-normal-state phase transition in overdoped $\mathrm{Bi}_{2} \mathrm{Sr}_{2} \mathrm{Ca}_{0.92} \mathrm{Y}_{0.08} \mathrm{Cu}_{2} \mathrm{O}_{8+\delta}$. Phys. Rev. B 83, 064519 (2011).

45. Zhao, L. et al. Quantitative determination of Eliashberg function and evidence of strong electron coupling with multiple phonon modes in heavily overdoped (Bi, $\mathrm{Pb})_{2} \mathrm{Sr}_{2} \mathrm{CuO}_{6+\delta}$. Phys. Rev. B 83, 184515 (2011).

46. Bianconi, A. et al. Determination of the local lattice distortions in the $\mathrm{CuO}_{2}$ plane of $\mathrm{La}_{1.85} \mathrm{Sr}_{0.15} \mathrm{CuO}_{4}$. Phys. Rev. Lett. 76, 3412-3415 (1996).

47. Zhao, G. M., Hunt, M. B., Keller, H. \& Muller, K. A. Evidence for polaronic supercarriers in the copper oxide superconductors $\mathrm{La}_{2-} \mathrm{Sr}_{\mathrm{x}} \mathrm{CuO}_{4}$. Nature 385, 236-239 (1997).

48. Lucy, L. B. An iterative technique for the rectification of observed distributions. Astron. J. 79, 745-754 (1974).

\section{Acknowledgements}

This work was supported by Berkeley Lab's programs on "Quantum Materials" and "Ultrafast Materials" funded by the US Department of Energy, Office of Science, Office of Basic Energy Sciences, Materials Sciences and Engineering Division, under contract no. DE-AC02-05CH11231.

\section{Author contributions}

W.Z., C.L.S. and T.L.M. carried out the measurements on Bi2212. C.H., C.L.S. and W.Z took the measurement on Bi2201. W.Z., C.H., C.L.S. and T.L.M. were responsible for data analysis. Bi2212 samples were prepared by H.E. Bi2201 sample was prepared by K.K., T.A. and Y.K. A.L. was responsible for the experimental planning and infrastructure. All authors contributed to the interpretation and writing of the manuscript.

\section{Additional information}

Supplementary Information accompanies this paper at http://www.nature.com/ naturecommunications

Competing financial interests: The authors declare no competing financial interests.

Reprints and permission information is available online at http://npg.nature.com/ reprintsandpermissions/

How to cite this article: Zhang, W. et al. Ultrafast quenching of electron-boson interaction and superconducting gap in a cuprate superconductor. Nat. Commun. 5:4959 doi: 10.1038/ncomms5959 (2014). 